\title{
COMPOSIÇÃO FLORÍSTICA E USOS MÚLTIPLOS DE ARBÓREAS EM SISTEMAS AGROFLORESTAIS EM RONDÔNIA
}

\author{
Mirian Gusmão ${ }^{1}$
}

Emanuel Maia²

Anna Frida Hatsue Modro²

\section{INTRODUÇÃO}

O tradicional modelo de colonização da Amazônia legal, resultado de políticas de ocupação desenvolvimentista na região, tem desencadeado um intenso desflorestamento e extinção de processos ecossistêmicos e sociais complexos (TURNER et al., 2001; FEARNSIDE, 2003; ALENCAR et al., 2004). A área cumulativa desmatada na Amazônia legal brasileira não é distribuída homogeneamente, mas concentrada ao longo do denominado "arco do desmatamento" (FEARNSIDE, 2005; FERREIRA et al., 2005) que abrange os estados do Pará, Mato Grosso, Rondônia, Maranhão e Tocantins e concentram o maior número de municípios, com áreas desmatadas superior a 80\% (RIVERO et al., 2009).

No momento em que a pressão sobre a floresta amazônica passou a ser mais evidente causando sérios problemas ambientais, programas de sensibilização e o foco das agências de desenvolvimento que atuam na Amazônia, passaram a analisar opções de sistemas de cultivos menos impactantes para a região (SILVA, 2013). Entre estas opções, os sistemas agroflorestais (SAF) têm sido muito utilizados como opção para exploração agropecuária, aumentando a diversificação de produtos e possibilitando a manutenção de processos ecossistêmicos (VIEIRA et al., 2007; JOSÉ, 2009; WILSON; LOVELL, 2016). Nestes sistemas, é obrigatório o uso

1 Programa de Pós-Graduação em Ciências Ambientais, Universidade Federal de Rondônia UNIR, Campus Rolim de Moura, Av. Norte Sul, 7300, Nova morada, CEP: 76940-000, e-mail: mirian.engflorestal@gmail.com;

2 Laboratório de Horticultura Tropical e Apicultura, Universidade Federal de Rondônia UNIR, Campus Rolim de Moura, Av. Norte Sul, 7300, Nova Morada, CEP: 76940-000, e-mail: emanuel@unir.br; anna.frida@unir.br 
de plantas arbóreas e/ou arbustivas em conjunto com espécies agrícolas e forrageiras com ou sem a presença animal (SANCHEZ, 1995; ABDO et al., 2008).

Os SAF auxiliam a evitar e reverter a degradação do meio ambiente, ao mesmo tempo em que proporcionam inúmeros serviços e bens ecossistêmicos (JOSÉ, 2009; MEYER, 2011) além de serem uma prática tradicional de cultivo para a agricultura familiar (SANCHEZ, 1995). Neste sentido, estes sistemas oferecem diversificação de produtos, benefícios e serviços ambientais, resultados da incorporação de um conjunto de técnicas de base agroecológica (VIEIRA et al., 2009; WILSON; LOVELL, 2016).

Os SAF têm sido vistos como alternativas de usos de espaços agrícolas, especialmente para regiões tropicais, por aumentar a diversificação de espécies cultivadas e a sustentabilidade de agrossistemas em seus aspectos agronômicos, sociais, econômicos e ecológicos, com variadas possibilidades de uso de espécies florestais (PAULA; PAULA, 2003; CASTRO et al., 2009). Neste sentido, considerando as unidades de produção familiar, destaca-se o potencial de usos múltiplos que o agroecossistema ou que determinadas espécies botânicas assumem, resultando em benefícios, produtos e serviços tangíveis e intangíveis (JERNECK; OLSSON, 2013; WILSON; LOVELL, 2016).

A variação na composição do componente arbóreo encontrada em agroflorestas na Amazônia pode apontar a importância destes sistemas como áreas de múltiplos usos, como também podem indicar o seu potencial para a conservação ambiental. Neste sentido, estudos realizados por Rodrigues (2005) e Silva (2013) relatam a riqueza botânica em SAF variando entre 50 a 89 espécies, com o índice de diversidade (Shannon-Weaver) entre 0,40 a 2,85. Estudos analíticos desta natureza, permitem comparações dentro e entre a composição florística no espaço e no tempo, além de gerar informações sobre a riqueza e diversidade de espécies, servindo de base para trabalhos posteriores, fundamentando teorias e hipóteses, além de poderem auxiliar na definição de modelos mais eficientes para a implantação e manejo destas áreas (CHAVES et al., 2013).

Entretanto, a falta de estudos e de definição de modelos sobre esses agroecossistemas tem reduzido o potencial de seu uso de forma mais sustentável (SANTOS et al., 2004). Sendo assim, esta 
pesquisa destaca a composição florística e o potencial de usos múltiplos tangíveis de espécies arbóreas cultivadas em sistemas agroflorestais na Amazônia Ocidental.

\section{PROTOCOLO EXPERIMENTAL}

Os dados foram coletados, entre o período de agosto de 2014 e julho de 2015, em três sistemas agroflorestais de bases agroecológicas, típicos de cultivo do cupuaçuzeiro (Theobroma grandiflorum), no município de Rolim de Moura - Rondônia (Figura 1). O município é um dos polos regionais e destaca-se por apresentar uma das maiores densidades populacionais na zona rural, associada a um dos maiores índices de desmatamento do estado (CAVALHEIRO et al., 2015).

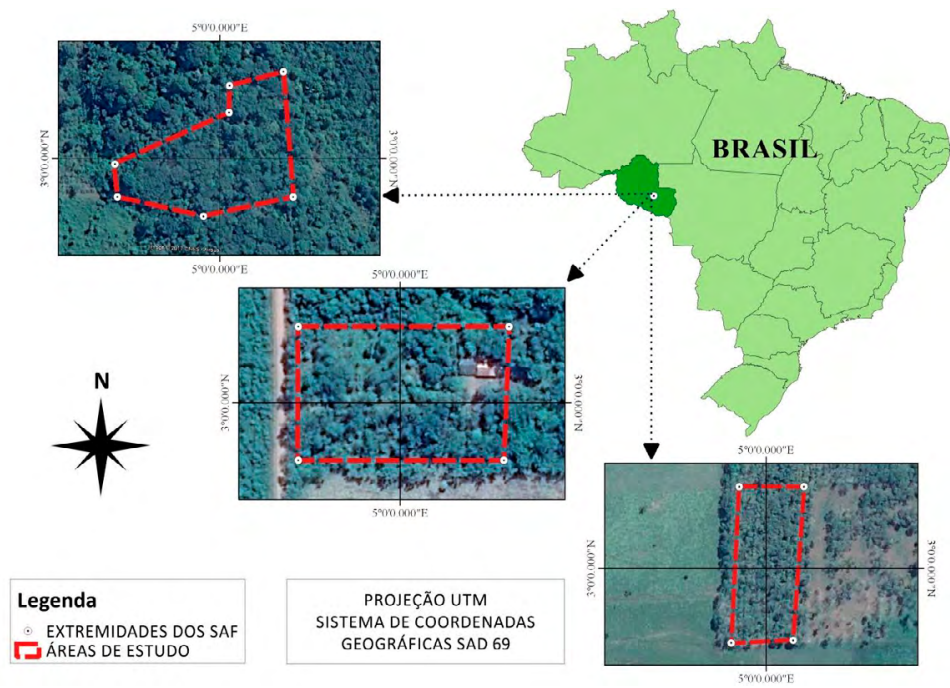

Figura 1. Localização de três Sistemas Agroflorestais estudados na região Central de Rondônia, Rolim de Moura, 2014-2015

A escolha das áreas foi realizada a partir da identificação de grupos agroflorestais com fitofisionomias semelhantes. Assim, após a identificação destes grupos, foram definidos três sistemas representativos como amostragem. Os sistemas possuem uma área de aproximadamente um hectare cada, com distintas histórias de 
implantação e manejo. O SAF 1 está localizado na Linha 172, km 13,5 ao norte, Sítio Dois Irmãos (S $11^{\circ} 36^{\prime} 05.2^{\prime \prime}$, W $061^{\circ} 52^{\prime} 13.4^{\prime \prime}$ ), este sistema data de aproximadamente 25 anos de idade, onde 0 cupuaçuzeiro foi introduzido em um fragmento de floresta sem espaçamento padronizado, os tratos culturais são escassos favorecendo a regeneração natural. O SAF 2 está localizado na Linha 168, km 1,5 sul, Sítio Vital Brasil (S 11 44' 24.85”, W 061 55' 24.66”), foi implantado há 15 anos, consorciando várias espécies frutíferas e madeireiras, neste sistema os tratos culturais são frequentes. $\mathrm{O}$ SAF 3 localiza-se na Linha 172, km 5,0 ao norte (S $11^{\circ} 40^{\prime} 45.24^{\prime \prime}$, W 061 53' 32.67"), foi implantado há cerca de 22 anos, onde o cupuaçuzeiro foi introduzido com espaçamento padronizado em um antigo seringal, os tratos culturais são frequentes.

$A$ vegetação característica da região é do tipo Floresta Ombrófila Aberta Submontana (IBGE, 2012), o solo é classificado como Latossolo vermelho-escuro eutrófico associado a um Latossolo vermelho-amarelo distrófico (SIPAM, 2006). A altitude média está entre 200 a 300 m, e segundo a classificação de Köppen-Geiger, o clima é do tipo Am - Clima Tropical Úmido com temperatura média entre 24 a 26 드, precipitação anual média de 2.200 a $2.500 \mathrm{~mm}$, e umidade relativa do ar oscilando em torno de $85 \%$ no período chuvoso, entre outubro a maio (ALVARES et al., 2013).

\section{Amostragem e Coleta dos Dados}

Para a escolha das áreas observou-se a presença do cupuaçuzeiro, sendo este o principal gerador de trabalho e renda em SAF na região. Foi realizado o censo das áreas, totalizando três hectares inventariados com mensuração e identificação ao menor nível taxonômico de todos os indivíduos com diâmetro maior ou igual a $5 \mathrm{~cm}$ na altura de 1,30 $\mathrm{m}$ do solo, sendo as espécies inclusas neste critério numeradas por placas metálicas (SILVA et al., 2005).

A identificação botânica foi efetuada mediante auxílio de profissionais especialistas ou pelo método comparativo através de consultas à herbários, chaves de identificação e por meio de literatura especializada. O sistema de classificação adotado foi o proposto pelo Angiosperm Phylogeny Group (APG III, 2009). Foram preenchidas fichas dendrológicas e coletados três exemplares de 
cada espécime, registrando-se o nome comum de cada uma de acordo com o conhecimento do agricultor. As amostras foram submetidas à secagem em estufa e incorporadas ao acervo do Laboratório de Horticultura Tropical da Universidade Federal de Rondônia - LaHorTA. As espécies comuns em pelo menos dois sistemas, foram classificadas, de acordo com a literatura, quanto ao seu potencial de uso múltiplo, em: madeireiro (MA); alimentação humana (AH); alimentação animal (AA); medicinal (ME); cosmético (CO); lenha/carvão (LC); ornamental (OR) e; quanto à produção de látex (LA).

\section{Análise de Dados}

A composição florística foi analisada considerando a ocorrência das espécies arbóreas nas áreas amostradas. Como indicadores de heterogeneidade foi calculado o índice de diversidade de Shannon-Weaver $\left(H^{\prime}\right)$ e a Equabilidade de Pielou ( $\left.J^{\prime}\right)$. A comparação entre os sistemas que expressa à semelhança entre ambientes baseando-se no número de espécies comuns, foi feita por meio do índice de similaridade de Jaccard (SJ) (MAGURRAN, 1988). Os parâmetros fitossociológicos da estrutura horizontal (Densidade, Frequência, Dominância e Valor de Cobertura) foram estimados como proposto por Mueller-Dombois e Ellenberg (1974).

\section{RESULTADOS E DISCUSSÃO}

Foram amostrados 2.319 indivíduos, compreendendo 113 espécies, distribuídas em 43 famílias botânicas. As famílias com maior número de espécies ( $\geq 6$ ) em ordem decrescente, foram: Fabaceae, Lauraceae, Moraceae, Annonaceae, Apocynaceae e Malvaceae, que, juntas representam $70 \%$ das espécies dos sistemas (Figura 2). Os SAF da região apresentaram maior riqueza de espécies e indivíduos em sua composição, se comparado a outros sistemas da Amazônia Ocidental (SILVA, 2013). 


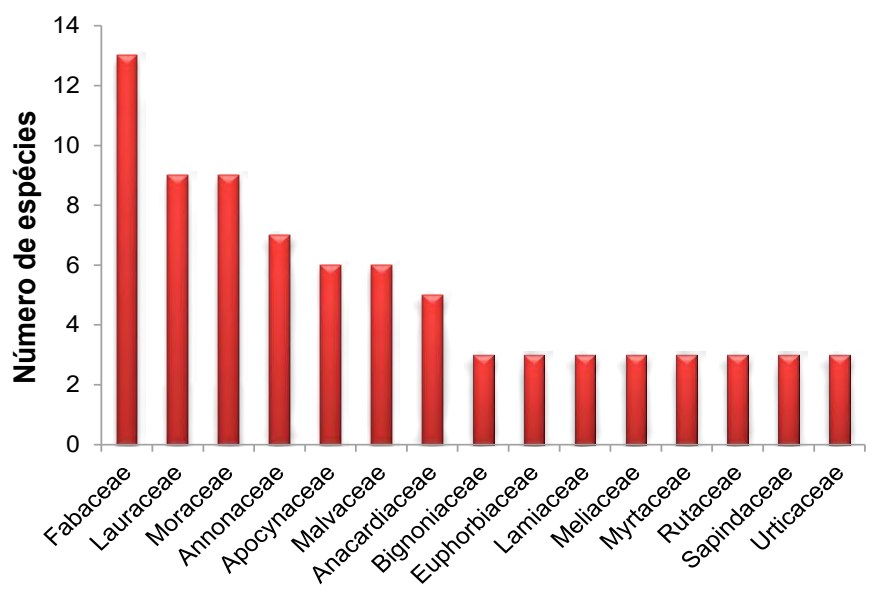

Famílias botânicas

Figura 2. Famílias com maior riqueza de espécies ( $\geq 3$ ) amostradas em três Sistemas Agroflorestais na região Central de Rondônia, Rolim de Moura, RO. 2014-2015

A Fabaceae mostrou-se a família com maior riqueza de espécies nos agrossistemas. Esta também tem sido apontada como a família com a maior riqueza em outros sistemas agroflorestais (SANTOS et al., 2004; RODRIGUES, 2005; FERNANDES et al., 2010; SALOMÃO et al., 2011). As espécies desta família destacam-se pela capacidade de associação com bactérias fixadoras de nitrogênio, o que permite a incorporação contínua de nitrogênio e a ciclagem de outros nutrientes ao solo (CAMPELLO et al., 2005). Estas plantas são recomendadas para recuperação de áreas degradadas, pois protegem o solo da erosão, favorecem a produção de matéria orgânica, e estimulam diversos processos químicos e biológicos do solo, melhorando sua fertilidade (BERTONI; LOMBARDI NETO, 2012). A introdução destas espécies em sistemas de recomposição florestal é considerada como uma técnica de baixo custo de implantação e manutenção (NOGUEIRA et al., 2012) e por suas vantagens, desempenha fundamental papel nos SAF.

O SAF 1 apresentou a maior riqueza florística seguida pelo SAF 2 e o SAF 3 (Tabela 1). Estes sistemas apresentaram maior abundância de espécie, gênero, família e em número de indiví- 
duos se comparados à estudos realizados na Amazônia brasileira (RODRIGUES, 2005; VIEIRA et al., 2007).

\begin{tabular}{ccccc}
\hline $\begin{array}{c}\text { Sistemas Agroflo- } \\
\text { restais }\end{array}$ & $\begin{array}{c}\text { Número de } \\
\text { Famílias }\end{array}$ & $\begin{array}{c}\text { Número de } \\
\text { Gênero }\end{array}$ & $\begin{array}{c}\text { Número de } \\
\text { Espécie }\end{array}$ & $\begin{array}{c}\text { Abundância (ind. } \\
\text { ha-1) }\end{array}$ \\
\hline 1 & 43 & 66 & 84 & 1.169 \\
\hline 2 & 19 & 29 & 33 & 489 \\
\hline 3 & 15 & 19 & 19 & 661 \\
\hline
\end{tabular}

Tabela 1. Composição florística de três Sistemas Agroflorestais da região Central de Rondônia, Rolim de Moura, RO, 2014-2015

A distribuição das espécies nos sistemas foi heterogênea (Tabela 2), o que pode ser atribuído aos diferentes manejos aplicados, a idade dos sistemas e principalmente, ao histórico de implantação de cada área. Isto pode ser observado, por exemplo, pela frequência da seringueira e do cupuaçu em todos os SAF, visto que são plantas de interesse comercial aos agricultores.

\begin{tabular}{|c|c|c|}
\hline Família/Espécie & Nome Comum & SAF \\
\hline \multicolumn{3}{|l|}{ Achariaceae } \\
\hline Lindackeria paludosa (Benth.) Gilg & Farinha seca & 1 \\
\hline \multicolumn{3}{|l|}{ Anacardiaceae } \\
\hline Anacardium occidentale L. & Caju & 1,2 \\
\hline Astronium lecointei Ducke & Aroeira & 2 \\
\hline Mangifera indica $\mathrm{L}$. & Mangueira & 1,2 \\
\hline Spondias mombin L. & Cajá & 2 \\
\hline Spondias purpurea $\mathrm{L}$. & Seriguela & 2 \\
\hline \multicolumn{3}{|l|}{ Annonaceae } \\
\hline contunuAnnona sp. & Anona & 3 \\
\hline Guatteria sp. & Pindaíba Preta & 1 \\
\hline Annonaceae sp. & - & 1 \\
\hline Rollinia sp. & Pinha da Mata & 1 \\
\hline Unonopsis sp. & Envira & 1 \\
\hline Xylopia sericea A. St.-Hil & Pindaíba Branca & 1 \\
\hline Xylopia sp. & Envira & 1 \\
\hline
\end{tabular}




\begin{tabular}{|c|c|c|}
\hline Apocynaceae & & \\
\hline Aspidosperma polyneuron Müll.Arg. & Peroba Rosa & 2 \\
\hline Aspidosperma sp. 1 & Peroba da Terra Amarela & 1 \\
\hline Aspidosperma sp. 2 & Peroba Mica & 2 \\
\hline Geissospermum laeve (Vell.) Miers & Pau Pereira & 1,3 \\
\hline Himatanthus articulatus (Vahl) Woodson & Sucuba & 1 \\
\hline Macoubea sprucei (Müll.Arg.) Markgr. & Pepino da Mata & 1 \\
\hline \multicolumn{3}{|l|}{ Araliaceae } \\
\hline $\begin{array}{l}\text { Schefflera morototoni (Aubl.) Maguire } \\
\text { et al. }\end{array}$ & Mandiocão & 1,3 \\
\hline \multicolumn{3}{|l|}{ Arecaceae } \\
\hline $\begin{array}{l}\text { Astrocaryum aculeatissimum (Schott) } \\
\text { Burret }\end{array}$ & Palmeira Brejaúva & 1,3 \\
\hline Arecaceae sp. & Palmeira & 1 \\
\hline \multicolumn{3}{|l|}{ Bignoniaceae } \\
\hline Handroanthus roseo-albus (Ridl.) Mattos & Ipê Branco & 1 \\
\hline Handroanthus Serratifolius (Vahl.) S. Grose & Ipê Amarelo & 1 \\
\hline Jacaranda copaia (Aubl.) D. Don & Caroba & 1,3 \\
\hline \multicolumn{3}{|l|}{ Burseraceae } \\
\hline Protium sp. & Breu & 1 \\
\hline Tetragastris altissima (Aubl.) Swart & Breu-manga & 2 \\
\hline \multicolumn{3}{|l|}{ Cannabaceae } \\
\hline Trema micrantha (L.) Blume & Curindiba (Grandiúva) & 2 \\
\hline \multicolumn{3}{|l|}{ Caryocaraceae } \\
\hline Caryocar sp. & Pequi & 1 \\
\hline \multicolumn{3}{|l|}{ Chrysobalanaceae } \\
\hline Conepia sp. & Castanha de Cutia & 1 \\
\hline Licania sp. & Pintadinha & 1 \\
\hline Família/Espécie & Nome Comum & SAF \\
\hline \multicolumn{3}{|l|}{ Combretaceae } \\
\hline Buchenavia sp. & Tanimbuca & 1 \\
\hline \multicolumn{3}{|l|}{ Elaeocarpaceae } \\
\hline Sloanea guianensis (Aubl.) Benth. & Urucurana & 1 \\
\hline
\end{tabular}




\begin{tabular}{|c|c|c|}
\hline Euphorbiaceae & & \\
\hline Conceveiba martiana Baill. & Canela de Cutia & 1 \\
\hline $\begin{array}{l}\text { Hevea brasiliensis (Willd. ex A.Juss.) Müll. } \\
\text { Arg. }\end{array}$ & Seringueira & $1,2,3$ \\
\hline Mabea pulcherrima Müll.Arg. & Cariúba & 2 \\
\hline \multicolumn{3}{|l|}{ Fabaceae } \\
\hline Dipteryx sp. & Cumaru & 1 \\
\hline \multicolumn{3}{|l|}{ Fabaceae-Caesalpinioideae } \\
\hline Apuleia leiocarpa (Vogel) J.F.Macbr. & Garapa & 1 \\
\hline $\begin{array}{l}\text { Chamaecrista adiantifolia (Benth.) H.S.Ir- } \\
\text { win \& Barneby }\end{array}$ & Baginha & 1 \\
\hline Swartzia cf. grandifolia Benth & Angelim Amargoso & 1,2 \\
\hline \multicolumn{3}{|l|}{ Fabaceae-Faboideae } \\
\hline Dalbergia sp. & - & 1 \\
\hline Luetzelburgia sp. & Guaiçara & 1 \\
\hline \multicolumn{3}{|l|}{ Fabaceae-Mimosoideae } \\
\hline Enterolobium sp. & Timburí & 1 \\
\hline $\begin{array}{l}\text { Enterolobium schomburgkii (Benth.) } \\
\text { Benth. }\end{array}$ & Baginha Vermelha & 1,3 \\
\hline Hymenolobium sp. 1 & Angelim Pedra & 1 \\
\hline Hymenolobium sp. 2 & Angelim Rosa & 1 \\
\hline Inga edulis Mart. & Ingá & 1 \\
\hline Parkia sp. & Angelim & 1,2 \\
\hline $\begin{array}{l}\text { Schizolobium parahyba var. amazonicum } \\
\text { (Huber ex Ducke) Barney }\end{array}$ & Bandarra & 2,3 \\
\hline \multicolumn{3}{|l|}{ Lamiaceae } \\
\hline Aegiphila integrifolia (Jacq.) Moldenke & Tamanqueiro & 1 \\
\hline Tectona grandis L. F. & Teca & 2 \\
\hline Vitex polygama Cham. & Maria Preta & 1 \\
\hline \multicolumn{3}{|l|}{ Lauraceae } \\
\hline Lauraceae sp .1 & - & 3 \\
\hline Lauraceae sp. 2 & - & 1 \\
\hline Mezilaurus itauba (Meisn.) Taub. ex Mez. & Itaúba & 2,3 \\
\hline Nectandra sp. 1 & Canela & 3 \\
\hline
\end{tabular}




\begin{tabular}{|c|c|c|}
\hline Nectandra sp. 2 & Canela & 1 \\
\hline Nectandra sp. 3 & Canela Preta & 1 \\
\hline Ocotea cf. diospyrifolia (Meisn.) Mez & Canela Amarela & 1 \\
\hline Ocotea sp. & Canela & 1 \\
\hline Persea americana Mill. & Abacate & 1,2 \\
\hline \multicolumn{3}{|l|}{ Lecythidaceae } \\
\hline Bertholletia excelsa Bonpl. & Castanha-do-brasil & 1,2 \\
\hline Couratari sp. & Imbirema & 1 \\
\hline \multicolumn{3}{|l|}{ Lythraceae } \\
\hline Physocalymma scaberrimum Pohl & Itaúba de Capoeira & 1 \\
\hline \multicolumn{3}{|l|}{ Malpighiaceae } \\
\hline Byrsonima sp. & Murici da Mata & 1 \\
\hline Malpighia glabra $\mathrm{L}$. & Acerola & 2 \\
\hline \multicolumn{3}{|l|}{ Malvaceae } \\
\hline Ceiba pentandra (L.) Gaertn. & Sumaúma & 1 \\
\hline Ceiba speciosa ( A. St. Hill ) Ravenna & Paineira & 2 \\
\hline Eriotheca gracilipes (K.Schum.) A.Robyns & Paineirinha & 2 \\
\hline Sterculia sp. & Embira Quiabo & 1 \\
\hline $\begin{array}{l}\text { Theobroma grandiflorum (Willd. ex } \\
\text { Spreng.) K.Schum. }\end{array}$ & Cupuaçu & $1,2,3$ \\
\hline Theobroma speciosum Willd. ex Spreng. & Cacau Nativo & 1 \\
\hline \multicolumn{3}{|l|}{ Melastomataceae } \\
\hline Bellucia grossularioides (L.) Triana & Araça-de-Anta & $1,2,3$ \\
\hline \multicolumn{3}{|l|}{ Meliaceae } \\
\hline Guarea sp. & - & 2 \\
\hline Trichilia sp. 1 & - & 1 \\
\hline Trichilia sp. 2 & - & 1 \\
\hline \multicolumn{3}{|l|}{ Meliaceae } \\
\hline Cedrela fissilis Vell. & Cedro Rosa & 1 \\
\hline \multicolumn{3}{|l|}{ Moraceae } \\
\hline Helicostylis scabra (J.F.Macbr.) C.C.Berg & Inharé & 1 \\
\hline Perebea mollis (Poepp. \& Endl.) Huber & Pama & 2 \\
\hline
\end{tabular}




\begin{tabular}{|c|c|c|}
\hline Artocarpus altilis (Parkinson) Fosberg & Fruta Pão & 2 \\
\hline Artocarpus heterophyllus Lam. & Jaca & 2 \\
\hline Bagassa guianensis Aubl. & Garrote & 1 \\
\hline Bagassa sp. & Garrote & 1 \\
\hline Ficus sp. & Figueira Mata-Pau & 1 \\
\hline Maclura cf. tinctoria (L.) D.Don ex Steud. & Moreira & 3 \\
\hline Maquira sp. & Cega Corrente & 1,3 \\
\hline \multicolumn{3}{|l|}{ Myrtaceae } \\
\hline Eugenia sp. & Orvalha & 2 \\
\hline Myrtaceae sp. & - & 1 \\
\hline Syzygium jambos (L.) Alston & Jambo Branco & 1,2 \\
\hline \multicolumn{3}{|l|}{ Peraceae } \\
\hline Pera sp. & Pera & 1,3 \\
\hline \multicolumn{3}{|l|}{ Polygonaceae } \\
\hline Coccoloba sp. & - & 1 \\
\hline \multicolumn{3}{|l|}{ Quinaceae } \\
\hline Quiina amazonica A.C.Sm. & - & 1 \\
\hline \multicolumn{3}{|l|}{ Rhamnaceae } \\
\hline Colubrina glandulosa G.Perkins & Sobrasil & 2 \\
\hline \multicolumn{3}{|l|}{ Rubiaceae } \\
\hline Rubiaceae sp. & - & 1 \\
\hline \multicolumn{3}{|l|}{ Rutaceae } \\
\hline Citrus latifolia Tanaka, cv. Tahiti & Limão Tahiti & 2 \\
\hline Citrus sp. & Mexirica & 2 \\
\hline Metrodorea flavida K. Krause & Pirarara Branca & 1 \\
\hline \multicolumn{3}{|l|}{ Salicaceae } \\
\hline Salicaceae sp. 1 & - & 3 \\
\hline \multicolumn{3}{|l|}{ Sapindaceae } \\
\hline Dilodendron bipinnatum Radlk. & Maria Pobre & 1 \\
\hline Sapindaceae sp. & - & 3 \\
\hline Toulicia cf. guianensis Aubl. & Maracatiara & 1 \\
\hline
\end{tabular}




\begin{tabular}{|c|c|c|}
\hline Sapotaceae & & \\
\hline Pouteria pachycarpa Pires & Quari quara & 1 \\
\hline Pouteria sp. & - & 1 \\
\hline \multicolumn{3}{|l|}{ Simaroubaceae } \\
\hline Simarouba amara Aubl. & Caixeta & 1 \\
\hline \multicolumn{3}{|l|}{ Siparunaceae } \\
\hline Siparuna guianensis Aubl. & Siparuna & 1,3 \\
\hline Solicaceae & & 1 \\
\hline Casearia sp. & Goiaba de Porcão & 1 \\
\hline \multicolumn{3}{|l|}{ Urticaceae } \\
\hline Cecropia engleriana Snethl. & Embaúba & 1,2 \\
\hline Cecropia sp. & Embaúba & 1 \\
\hline Pourouma sp. & Manduvi & 1 \\
\hline \multicolumn{3}{|l|}{ Violaceae } \\
\hline Rinorea sp. & - & 1 \\
\hline \multicolumn{3}{|l|}{ Morfotipo } \\
\hline Morfotipo 1 & - & 1 \\
\hline Morfotipo 2 & - & 1 \\
\hline Morfotipo 3 & - & 1 \\
\hline
\end{tabular}

Tabela 2. Composição florística (famílias e espécies) de três Sistemas Agroflorestais localizados na região Central de Rondônia, Rolim de Moura, RO, 2014-2015

A seringueira teve uma frequência nos sistemas de $32 \%$, o que está atribuída a antigos seringais na região, implantados por estímulo de financiamentos governamentais para a produção de látex (D'AGOSTINI et al., 2013). O cupuaçuzeiro é a espécie frutífera com maior abundância nos sistemas com representatividade de $26 \%$, e esta espécie tem sido a mais frequente em citações de pesquisas agroflorestais na Amazônia (BRIENZA JÚNIOR et al., 2009; CASTRO et al., 2009; SILVA, 2013) e pode-se deduzir que esta combinação é comum nos sistemas agroflorestais da região amazônica.

O araçá-de-anta (Bellucia grossularioides) apesar de ser comum em todas as áreas, apresenta abundância de apenas 0,6\%, considerando que não é de interesse comercial e nem alimentar aos produtores, entretanto sua frequência é explicada por ser de 
ocorrência típica de áreas nativas na região (REFLORA, 2016).

Outras espécies arbóreas ocorrentes em pelo menos dois sistemas, foram: abacate (Persea americana), angelim (Parkia sp.), angelim amargoso (Swartzia cf. grandifolia), baginha vermelha (Enterolobium schomburgkii), bandarra (Schizolobium parahyba var. amazonicum), caju (Anacardium occidentale), caroba (Jacaranda copaia), castanha-do-brasil (Bertholletia excelsa), cega corrente (Maquira sp.), embaúba (Cecropia engleriana), itaúba (Mezilaurus itauba), jambo branco (Syzygium jambos), mangueira (Mangifera indica), palmeira brejaúva (Astrocaryum aculeatissimum), pau pereira (Geissospermum laeve), pera (Pera sp.) e siparuna (Siparuna guianensis). As demais espécies (81,4\%) ocorreram em apenas um dos sistemas, sendo observado que, grande parte das espécies ocorrentes nos SAF estudados também compõem outros sistemas na Amazônia (SILVA, 2013).

A maior diversidade florística e equitabilidade foi encontrado para o SAF 1, seguido do SAF 2 e por último o SAF 3 (Tabela 3). Essa constatação foi esperada, pois a equitabilidade é diretamente proporcional à diversidade e, antagônico à dominância (UHL; MURPHY, 1981). Teoricamente, esse valor indica que seria necessário o incremento de $39 \%$ das espécies para o SAF 1 , de $40 \%$ para o SAF 2 e de $73 \%$ para o SAF 3 para atingir, assim, a diversidade máxima da comunidade vegetal (BROWER et al., 1998). O índice de diversidade dos SAF 1 e 2 está acima e a equitabilidade está abaixo dos valores encontrados em outras agroflorestas da Amazônia (SANTOS et al., 2004; RODRIGUES, 2005; SILVA, 2013).

\begin{tabular}{ccc}
\hline Sistemas Agroflorestais & H' $^{\prime}$ & $\mathbf{J}^{\prime}$ \\
\hline 1 & 2,70 & 0,61 \\
\hline 2 & 2,09 & 0,60 \\
\hline 3 & 0,80 & 0,27 \\
\hline
\end{tabular}

Tabela 3. Índices de diversidade de Shannon-Weaver $\left(\mathrm{H}^{\prime}\right)$ e Equitabilidade de Pielou ( $\left.J^{\prime}\right)$ de três Sistemas Agroflorestais localizados na região Central de Rondônia, Rolim de Moura, RO, 2014-2015

$O$ índice de similaridade mostra baixa semelhança entre os locais de estudo. Os SAF 1 e 3 apresentaram a maior proximidade, embora o valor do Índice de Jaccard (SJ) tenha sido baixo. As simi- 
laridades entre os SAF 2 e 3, e os SAF 1 e 2 foram ainda menores (Tabela 4). Valores maiores ou iguais a 0,5 indicam alta similaridade (KENT; COKER, 1992), assim, a similaridade encontrada entre as áreas pode ser considerada como baixa, indicando alta heterogeneidade florística dos sítios estudados. A similaridade entre os SAF se apresentou menor em relação a outros estudos (SANTOS et al., 2004; GAZEL FILHO et al., 2009; SILVA, 2013). A baixa proximidade da composição florística das áreas é comum em agroecossistemas tradicionais (PARDO-DE-SANTAYANA; MACÍA, 2015), no entanto, devido à heterogeneidade desta composição, estes sistemas podem representar paisagens rurais com grande importância para a conservação de espécies da flora e fauna regionais (WILSON; LOVELL, 2016).

\begin{tabular}{cccc}
\hline Sistemas Agroflorestais & $\mathbf{1}$ & $\mathbf{2}$ & $\mathbf{3}$ \\
\hline $\mathbf{1}$ & 1,00 & 0,09 & 0,12 \\
\hline $\mathbf{2}$ & - & 1,00 & 0,10 \\
\hline $\mathbf{3}$ & - & - & 1,00 \\
\hline
\end{tabular}

Tabela 4. Matriz de Similaridade Florística (Jaccard) em três Sistemas Agroflorestais da Região Central de Rondônia, Rolim de Moura, RO, 2014-2015

Os sistemas se apresentaram com potencial elevado de uso múltiplo, pois das espécies comuns em pelo menos dois sistemas, $62 \%$ possuem aproveitamento para alimentação animal e uso medicinal. Caso sejam adotados critérios de seleção considerando a diversificação botânica e o uso múltiplo de espécies, os sistemas agroflorestais são excelentes áreas para garantir a segurança alimentar e econômica das famílias de agricultores, visto que foram identificadas plantas de uso madeireiro (48\%); de alimentação humana, e uso ornamental (38\%); as utilizadas como cosméticos (29\%); aquelas aproveitadas para carvão ou produção de lenha (10\%) e ainda, aquelas com potencial para a produção de látex (5\%) (Figura 3).

É importante que seja considerado o potencial dessas espécies quanto a seus diferentes usos, no planejamento e manejo de SAF. Neste sentido, destaca-se a importância das fruteiras, pois podem contribuir na segurança alimentar e nutricional das famílias, além de seus frutos serem utilizados pela fauna e comercializados em 
mercados locais e regionais (SHANLEY; MEDINA, 2005; ALMEIDA et al., 2012).

Uma das avaliações do potencial florestal de um ecossistema é atribuída a importância econômica dos produtos que a mesma pode proporcionar à sociedade (GAMA et al., 2007). Sendo assim, entre as espécies mais frequentes nos três sistemas estudados neste trabalho, as que apresentaram maior potencial de uso foram a mangueira, a castanha-do-brasil e a seringueira, cada uma com cinco usos, seguidas pelo cupuaçu, caju, abacate, jambo branco, palmeira brejaúva e mandiocão, cada uma com quatro usos.

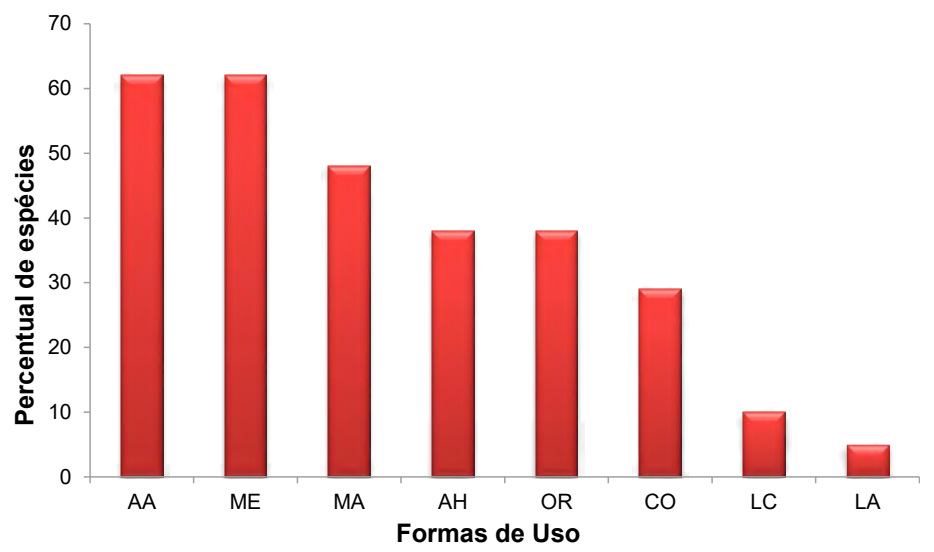

Figura 3. Principais formas de utilização de espécies mais representativas em três Sistemas Agroflorestais estudados na região Central de Rondônia, Rolim de Moura, RO, 2014-2015. Formas de uso: madeireiro (MA); alimentação humana (AH); alimentação animal (AA); medicinal (ME); cosmética (CO); lenha/carvão (LC); ornamental (OR) e quanto à produção de látex (LA)

A mangueira está entre as principais espécies frutíferas de clima tropical (FACHINELLO et al., 2008) originária da Ásia, especificamente da índia, tem ampla apreciação devido ao seu sabor, aroma e coloração bem característicos e atrativos, sendo preferencialmente consumida in natura. A maior parte da produção de seus derivados é por doces e geleias (CAMPOS et al., 2012), e é bastante utilizada na composição arbórea da paisagem rural. O chá da folha da mangueira é indicado para o tratamento de diarreia (MOREIRA et al., 2002) e o uso de garrafada proveniente de sua casca é utilizada para problemas do aparelho respiratório (SCHAR- 
DONG; CERVI, 2000; MONTELES; PINHEIRO, 2007). Da semente é extraída a manteiga que é um ingrediente cosmético funcional com grande potencial, contendo elevado teor de tocoferol, toesteróis e triterpenos (BHATTACHARYA; SHUKLA, 2002).

A castanha-do-brasil, considerada uma das espécies nativas mais valiosas da floresta amazônica de terra firme, é utilizada há várias gerações como fonte de alimentação e renda (COSTA et al., 2009). É uma espécie de uso múltiplo, pois produz a madeira e o fruto (amêndoa), este é muito apreciado no Brasil e no mundo, sendo um dos principais produtos de exportação da região amazônica, sua amêndoa é comerciada essencialmente in natura (SOUZA et al., 2008; ANGELO et al., 2013).

O fruto da castanha-do-brasil, também chamado "ouriço", pode pesar de $500 \mathrm{~g}$ a $1.500 \mathrm{~g}$. A amêndoa presente no interior da semente é rica em cálcio e fósforo, essenciais na alimentação infantil, possuindo elevado índice de selênio, magnésio e potássio, minerais importantes para o equilíbrio da saúde, com ação rejuvenescedora e energética (VILHENA, 2004; COSTA et al., 2009).

A castanheira é uma espécie rústica de crescimento rápido e madeira de boa qualidade, com rotações estimadas entre 30 e 40 anos e perspectivas de produção de madeira acima de $150 \mathrm{~m}^{3}$ ha ${ }^{-1}$ (ALMEIDA et al., 2012). Sua madeira é muito valorizada pelas indústrias sendo de ótima qualidade para construção civil interna leve, tábuas para assoalhos e paredes, painéis decorativos, forros, fabricação de compensados e embalagens (SOUZA et al., 2008). Tem grande potencial silvicultural para reflorestamentos com fins madeireiros (TONINI et al., 2005). Embora seja uma espécie protegida por lei, é recomendada para consórcios em áreas degradadas, pela boa forma do fuste e sua desrama natural, além de apresentar rusticidade, tolerância à luz e crescimento relativamente rápido (SOUZA et al., 2008).

A seringueira é natural da Região Amazônica, das onze espécies do gênero, a originária do Brasil, Hevea brasiliensis, é a que tem a maior capacidade produtiva com maior variabilidade genética (COSTA et al., 2001). Esta espécie possui múltiplos usos, desde a extração do látex, de grande importância histórica na região, utilizada na fabricação de pneumáticos e em centenas de artefatos de grande utilidade para a sociedade, até a produção de madeira e extração 
de óleo de suas sementes. Pesquisas têm sido direcionadas para o desenvolvimento de clones com dupla aptidão, contribuindo para agregação de renda extra, principalmente para os agricultores de base familiar (SOUZA, 2007).

A densidade da madeira seca ao ar oscila entre 0,56 a 0,65 g/ $\mathrm{cm}^{3}$. O teor de umidade da madeira recém-cortada se aproxima de 60 a 80\% (KILLMANN; HONG, 2000). No Brasil, a madeira da seringueira é obtida no final do seu ciclo produtivo de látex, sendo utilizada, tradicionalmente de forma quase exclusiva para fins energéticos (LARA PALMA, 2010). A madeira remanescente é extraída de plantas que não produzem mais látex, podendo ser utilizadas como combustível, celulose, na indústria de móveis, na fabricação de portas, janelas, formas para concreto armado, vigas, colunas, painéis de madeira compensada, energia, fabricação de tabuados, forros, caixotaria e painéis de cimento madeira (MAY; GONÇALVES, 1999; IAPAR, 2004; OKINO et al., 2004).

O cupuaçuzeiro normalmente cultivado no sub-bosque de agroflorestas é uma cultura importante para a região amazônica, apresenta grande potencial comercial, tanto para a produção de polpa da fruta, como para a utilização das suas sementes na indústria de alimentos e cosméticos (CASTRO et al., 2009; SILVA, 2013). É uma espécie nativa da Amazônia e está em processo de domesticação, seu cultivo é restrito aos estados da região norte e alguns países que fazem parte da Amazônia (KAMINSKI, 2006). Sua polpa possui alto valor econômico, sendo consumida como suco, néctar, iogurte, sorvete, creme, licor, torta, geleia, compota, biscoito, sorvete, e outros doces, que em sua maioria, são processados em pequenas escalas de produção, de forma artesanal, mas com alto valor econômico (COHEN; JACKIX, 2005). De suas sementes pode-se obter o cupulate, um produto semelhante ao chocolate, porém com menor concentração do estimulante cafeína e a manteiga é similar à do cacau, com a vantagem de ser obtida por um processo mais econômico, e pode também ser empregada na produção de cupulate em tabletes e na indústria de cosmético (KAMINSKI, 2006).

O caju, o abacate e o jambo branco são espécies frutíferas cultivadas em pelo menos dois dos três sistemas estudados, evidenciando a diversificação de produtos para uma maior segurança alimentar, nutricional e econômica das unidades de produção fa- 
miliares (MACÊDO, 2007). Neste sentido, nota-se a preferência dos agricultores por espécies permanentes, especialmente as frutíferas que possuem uma relação ecológica com a fauna e também, potencial de uso ornamental (FRANKE et al., 1998; VIEIRA et al., 2007).

A palmeira brejaúva, faz parte da família Arecaceae, que se destaca por ser uma das maiores do mundo, compreendendo 2.700 espécies distribuídas em 240 gêneros (LORENZI et al., 2010). Nessa família encontram-se espécies que dão suporte a fauna silvestre, através de suas folhas, polpa dos frutos e sementes (LIMA et al., 2003). Estudos mostram que o conhecimento e uso da palmeira brejaúva, são comuns em comunidades tradicionais brasileiras (LIMA et al., 1996; HANAZAKI; MIRANDA, 2006; MIRANDA; HANAZAKI, 2008). As palmeiras se destacam por serem amplamente utilizadas e exploradas comercialmente pela população humana devido aos produtos e subprodutos que podem ser obtidos, como óleo, amido, palmito, cera e fibras (SANTELLI et al., 2006; BÜTTOW et al., 2009), sendo consideradas um dos recursos vegetais mais importantes para o homem (MIRANDA et al., 2001). São também utilizadas como matéria prima na construção de casas, pontes e barcos (SANTELLI et al., 2006) ou utilizadas como plantas ornamentais (SOUZA; LORENZI, 2008).

O mandiocão pertence ao gênero Schefflera, somente na América tropical são conhecidas 205 espécies deste gênero (FRODIN et al., 2010), sendo que, 51 destas são nativas do Brasil (FIASCHI, 2015). A madeira é leve a moderadamente pesada, com densidade específica de 0,35 a $0,60 \mathrm{~g} / \mathrm{cm}^{3}$, tem como característica uma madeira esbranquiçada, com tonalidade acinzentada (LOUREIRO; LISBOA, 1979; OHASHI; LEÃO, 2005). Sua madeira é muito utilizada em carpintaria em geral, construções interiores, marcenaria, esquadrias, forros, caixas, engradados, lápis, palitos de fósforo, instrumentos musicais, brinquedos, compensados, celulose e papel. Esta espécie é indicada para recomposição de áreas alteradas, e devido a sua beleza e crescimento rápido são indicadas também para projetos paisagísticos (OHASHI; LEÃO, 2005), suas sementes são empregadas em artesanatos para confecção de colares, pulseiras e brincos (MACIEIRA et al., 2014). 


\section{CONSIDERAÇÕES FINAIS}

Constatou-se neste trabalho que, os sistemas agroflorestais em Rondônia são áreas diversificadas e com baixa similaridade florística, o que ressalta a importância desses sistemas para a conservação de espécies vegetais e animais. Esta heterogeneidade dos SAF reflete a relação sistêmica que ocorre nas unidades de produção familiar, onde cada área possui idade, história de implantação e manejo distintos. As áreas apresentam composições florísticas diferentes, sendo influenciadas, principalmente, pelo valor econômico das espécies, como o cupuaçuzeiro e a seringueira que são as plantas mais frequentes nestes sistemas. Entretanto, nota-se que, as espécies mais recorrentes nestas áreas apresentam múltiplos usos, destacando-se o potencial destas plantas para a alimentação animal e uso medicinal.

A seleção de espécies para a composição de SAF na região Amazônica, baseada em critérios de uso múltiplo pelas famílias de agricultores, apresenta uma estratégia promissora na diversificação, planejamento e manejo de agroflorestas, garantindo uma maior segurança alimentar, nutricional e econômica, além de potencializar a exploração de áreas cultivadas, proporcionando baixo impacto ambiental para regiões devastadas por ações antrópicas e com histórico de colonização recente, como a que apresenta na região central de Rondônia.

\section{REFERÊNCIAS}

ABDO, M.T.N.; VALERI, S.V.; MARTINSET, A.L.M. 2008. Sistemas agroflorestais e agricultura familiar: uma parceria interessante. Revista Tecnologia \& Inovação Agropecuária, São Paulo, 1: 50-59.

ALENCAR, A.; NEPSTAD, N.; MCGRATH, D.; MOUTINHO, P.; PACHECO, P.; DIAZ, M. D. C. V.; BRITALDO FILHO, S. 2004. Desmatamento na Amazônia: indo além da emergência crônica. Instituto de Pesquisa Ambiental da Amazônia, Manaus, 89p.

ALMEIDA, L.S.; GAMA, J.R.V.; OLIVEIRA, F.A.; CARVALHO, J.O.P.; GONÇALVES, D.C.M.; ARAÚJO, G.C. 2012. Fitossociologia e uso múltiplo de espécies arbóreas em floresta manejada, Comunidade Santo Antônio, município de Santarém, Estado do Pará. Acta Amazonica, Manaus, 42: 185-194.

ALVARES, A.C.; STAPE J.L.; SENTELHAS, P.C.; GONÇALVES, J.L.M.; SPAROVEK, G. 
2013. Köppen's climate classification map for Brazil. Meteorologische Zeitschrift, 22: 711-728.

ANGELO, H.; POMPERMAYER, R.S.; ALMEIDA, N.A.; MOREIRA, J.M.M.A.P. 2013.0 custo social do desmatamento da Amazônia brasileira: o caso da castanha-do-brasil (Bertholletia excelsa). Ciência Florestal, Santa Maria, 23: 183-191.

APG III. An update of the Angiosperm Phylogeny Website, Version 13. 2009. Disponivel em: <http://www.mobot.org/MOBOT/research/APweb/>. Acesso em: 28/06/2015. BERTONI, J.; LOMBARDI NETO, F. 2012. Conservação do solo. 8va ed. Ícone: São Paulo, 432-355p.

BHATTACHARYA, K.; SHUKLA, V.K. 2002. Manteiga de manga em formulações cosméticas. Cosmet Toil, 117: 65-70.

BRIENZA JÚNIOR, S.; MANESCHY, R.Q.; MOURÃO JÚNIOR, M.; GAZEL FILHO, A.B.; YARED, J.A.G.; GONÇALVES, D.; GAMA, M.B. 2009. Sistemas agroflorestais na Amazônia brasileira: análise de 25 anos de pesquisas. Pesquisa Florestal Brasileira, 60: 67-76.

BROWER, J.E.; ZAR, J.H.; VAN ENDE, C.N. 1998. Field and laboratory methods for general ecology. 4 th. New York: WCB/McGraw, 273p.

BÜTTOW, M.V.; BARBIERI, R.L.; NEITZKE, R.S.; HEINDEN, G. 2009. Conhecimento tradicional associado ao uso de butiás (Butia spp., Arecaceae) no sul do Brasil. Revista Brasileira de Fruticultura, São Paulo, 31: 1069-1075.

CAMPELLO, E.F.C.; RESENDE, A.S.; FRANCO, A.A. 2005. Importância da fixação biológica de nitrogênio na recuperação de áreas degradadas e manejo de sistemas agroflorestais. In: Seminário Petrobrás de Experiências Florestais. Salvador. Anais... Salvador, 2005.

CAMPOS, H.G.; MARCZAK, L.D.F.; ZANCAN, K.; GURAK, P.D. 2012. Avaliação de parâmetros físico-químicos de doce de manga enriquecido com resíduos provenientes do processamento da polpa. Trabalho de Conclusão de Curso, Universidade Federal do Rio Grande do Sul, Porto Alegre, Rio Grande do Sul, 36p.

CASTRO, A.P.; FRAXE, T.J.P.; SANTIAGO, J.L.; MATOS, R.B.; PINTO, I.C. 2009. Os sistemas agroflorestais como alternativa de sustentabilidade em ecossistemas de várzea no Amazonas. Revista Acta Amazonica, Manaus, 39: 279-288.

CAVALHEIRO, W.C.S.; VENDRUSCOLO, J.; SANTOS, L. M. H.; SANTOS, A. M. 2015. Impacto da colonização na Zona da Mata Rondoniense, Amazônia Ocidental-Brasil. Revista Geográfica Venezolana, 56: 41-57.

CHAVES, A.D.C.G.; SANTOS, R.M.S.; SANTOS, J.O.; FERNANDES, A.A.; MARACAJÁ, P.B. 2013. A importância dos levantamentos florístico e fitossociológico para a conservação e preservação das florestas. Agropecuária Científica no Semiárido, Campina Grande, 9: 42-48.

COHEN, K.O.; JACKIX, M.N.H. 2005. Estudo do liquor de cupuaçu. Ciência e Tecnologia de Alimentos, Campinas, 25: 182-190. 
COSTA, J.R.; CASTRO, A.B.C.; WANDELLI, E.V.; CORAL, S.C.T. SOUZA, S.A.G. 2009. Aspectos silviculturais da castanha-do-brasil (Bertholletia excelsa) em sistemas agroflorestais na Amazônia Central. Acta Amazonica, Manaus, 39: 843-850.

COSTA, R.B. GONÇALVES, P.S.; ODALIA-RÍMOLIA, A.; ARRUDA, E.J. 2001. Melhoramento e conservação genética aplicados ao desenvolvimento local - o caso da seringueira (Hevea sp.). Revista Internacional de Desenvolvimento Local, Campo Grande, 1:51-58.

D’AGOSTINI, S.; BACILIERI, S.; VITIELLO, N.; HOJO, H.; BILYNSKYJ, M.C.V.; BATISTA FILHO, A.; REBOUÇAS, M.M. 2013. Ciclo econômico da borracha - Seringueira (Hevea brasiliensis (hbk) m. Arg). Páginas do Instituto Biológico, São Paulo, 9: 6-14.

FACHINELLO, J.C.; NACHTIGAL, J.C.; KERSTEN, E. 2008. Fruticultura: fundamentos e práticas. 1ra ed. Pelotas, Rio Grande do Sul, 183p.

FEARNSIDE, P.M. 2003. A floresta amazônica nas mudanças globais. 1ra ed. INPA: Manaus, Amazonas, 134p.

FEARNSIDE, P.M. 2005. Desmatamento na Amazônia brasileira: história, índices e consequências. Megadiversidade, Manaus, 1: 113-123.

FERNANDES, S.S.L.; PADOVAN, M.P.; PEREIRA, Z.V.; MOITINHO, M.R.; HEID, D.M. 2010. Fitossociologia do componente arbóreo de um sistema agroflorestal no Assentamento Lagoa Grande, Município de Dourados, MS. p.1-5. In: Seminário de Agroecologia de Mato Grosso do Sul, 3. Corumbá. Anais... Dourados, Embrapa Agropecuária Oeste, Corumbá, Embrapa Pantanal, Campo Grande, Embrapa Gado de Corte, 2010.

FERREIRA, L.V.; VENTICINQUE, E.; ALMEIDA, S. 2005. O desmatamento na Amazônia e a importância das áreas protegidas. Estudos Avançados, Manaus, 19: 167-166.

FIASCHI, P. 2015. Araliaceae. In: Lista de Espécies da Flora do Brasil. Jardim Botânico do Rio de Janeiro. Disponível em: <http://floradobrasil.jbri.gov. br/jabot/ floradobrasil/FB15660>. Acesso em: 01/08/2017.

FRANKE, I.L.; LUNZ, A.M.P.; AMARAL, E.F. 1998. Caracterização socioeconômica dos agricultores do grupo Nova União, Senador Guiomard Santos, Acre: ênfase para implantação de sistemas agroflorestais. Rio Branco: Embrapa-Cpaf, 1998. 39p.

FRODIN, D.G.; LOWRY II, P.P.; PLUNKETT, G.M. 2010. Schefflera (Araliaceae): taxonomic history, overview and progress. Plant Diversity and Evolution, Stuttgart, 128: 561-595.

GAMA, J.R.V.; SOUZA, A.L.; CALEGÁRIO, N.; LANA, G.C. 2007. Fitossociologia de duas fitocenoses de floresta ombrófila aberta no município de Codó, Estado do Maranhão. Revista Árvore, Viçosa, 31: 465-477.

GAZEL FILHO, A.B. YARED, J.A.G.; MOURÃO JÚNIOR, M.; SILVA, M.F.; BRIENZA JÚNIOR, S.; FERREIRA, G.; SILVA, P.T.E. 2009. Diversidade e similaridade entre a vegetação de quintais agroflorestais em Mazagão, AP. p.1-4. In: Congresso Brasileiro de Sistemas Agroflorestais, 7. Luziânia. Anais... Luziânia, Sociedade Brasileira de Sistemas Agroflorestais, 2009. 
HANAZAKI, N.; MIRANDA, T.M. 2006. Conhecimento etnobotânico sobre a flora nativa das regiões do Parque Estadual Carlos Botelho (PECB) e do Parque Estadual da Ilha do Cardoso (PEIC). In: Relatório Temático do Projeto Parcelas Permanentes, 4. Piracicaba. Anais... Piracicaba, O Homem no Contexto Florestal, 2006.

IAPAR - INSTITUTO AGRONÔMICO DO PARANÁ. 2004. O Cultivo da Seringueira (Hevea spp.). Secretaria de Estado da Agricultura e do Abastecimento, Londrina, PR, 2004. Disponível em: <http://www.iapar.br/arquivos/File/zip pdf/cultsering. pdf $>$. Acesso em: 01/08/2017.

IBGE - Instituto Brasileiro de Geografia e Estatística. 2012. Manual técnico da vegetação brasileira. Rio de Janeiro: IBGE. 92 p., 2012.

JERNECK, A.; OLSSON, L. 2013. More than trees! Understanding the agroforestry adoption gap in subsistence agriculture: Insights from narrative walks in Kenya. Journal of Rural Studies, 32: 114-125.

JOSÉ, S. 2009. Agroforestry for ecosystem services and environmental benefits: an overview. Agroforestry Systems, 76: 1-10.

KAMINSKI, P.E. 2006. O cupuaçu: usos e potencial para o desenvolvimento rural na Amazônia. Revista Cafeicultura, Minas Gerais, 2006. Disponível em: <revistacafeicultura.com.br/?mat=4238>. Acesso em: 01/08/2017.

KENT, M.; COKER, P. 1992. Vegetation Description and Analysis: a practical approach. London: Belhaven Press, 363p.

KILLMANN, W.; HONG, L.T. 2000. El caucho, el éxito de un subproducto agrícola. Unasylva, 51: 66-72.

LARA PALMA, H.A. 2010. Propriedades técnicas e utilização da madeira da seringueira. p. 1-18. In: Ciclo de Palestras sobre a Heveicultura Paulista, 7. São José do Rio Preto. Anais... São José do Rio Preto, 2010.

LIMA, R.X.; KUNIYOSHI, Y.S.; SILVA, S.M. 1996. Estudos etnobotânicos em comunidades continentais da área de proteção ambiental de Guaraqueçaba Paraná - Brasil. Dissertação de mestrado, Universidade Federal do Paraná, Curitiba, Paraná, 124p.

LIMA, S.E.; FELFILI, J.M.; MARIMON, S.B.; SCARIOT, A. 2003. Diversidade, estrutura e distribuição espacial de palmeiras em um cerrado sensu stricto no Brasil Central - DF. Revista Brasileira de Botânica, São Paulo, 26: 361-370.

LORENZI, H.; NOBLICK, L.; KHAN, F.; FERREIRA, E. 2010. Flora Brasileira: Arecaceae (Palmeiras). 1ra ed. Nova Odessa: Instituto Plantarum de Estudos da Flora, 368p.

LOUREIRO, A.A.; LISBOA, P.L.B. 1979. Madeiras do município de Aripuanã e suas utilidades (Mato Grosso). Acta Amazonica, Manaus, 9: 88p.

MACÊDO, J.L.V. 2007. Cultivo de fruteiras em sistemas agroflorestais. In: Encontro de Frutas Nativas do Norte e Nordeste do Brasil, 1. São Luis. Anais... São Luis, Frutas Nativas: Novos Sabores para o Mundo. 
MACIEIRA, A.P.; COSTA, C.C.; CARVALHO, L.T.; FIASCHI, P.; GOMES, J.I.; MARTINS-DA-SILVA, R.C.V.; MARGALHO, L.F. 2014. Conhecendo Espécies de Plantas da Amazônia: Morototó (Schefflera morototoni (Aubl.) Maguire, Steyerm. \& Frodin - Araliaceae). Belém: Embrapa Amazonia Oriental, 4p.

MAGURRAN, A.E. 1988. Ecological diversity and its measurement. New Jersey: Princeton University Press, 179p.

MAY, A.; GONÇALVES, P.S. 1999. Produtos complementares na exploração do seringal - Matéria técnica. Borracha Atual, São Paulo, 17-21.

MEYER, M. 2011. Sistemas agroflorestais na Zona da Mata: experiências, aprendizados e propostas para a sustentabilidade. In: Porro, R.; MICCOLLIS, A. (Org.) Políticas públicas para o desenvolvimento agroflorestal no Brasil. Belém: Icraf, p. 46-58.

MIRANDA, I.P.A.; RABELO, A.; BUENO, C.R.; BARBOSA, E.M.; RIBEIRO, M.N.S. 2001. Frutos de palmeiras da Amazônia. Manaus: Ministério de Ciência e Tecnologia, Instituto Nacional de pesquisa da Amazônia, 120p.

MIRANDA, T.M.; HANAZAKI, N. 2008. Conhecimento e uso de recursos vegetais de restinga por comunidades das ilhas do Cardoso (SP) e de Santa Catarina (SC), Brasil. Acta Botânica Brasilica, 22: 203-215.

MONTELES, R.; PINHEIRO, C.U.B. 2007. Plantas medicinais em um quilombo maranhense: uma perspectiva etnobotânica. Biologia e Ciências da Terra, 7, núm. 2, 38-48.

MOREIRA, R.C.T.; COSTA, L.C.B.; COSTA, R.C.S.; ROCHA, E.A. 2002. Abordagem Etnobotânica acerca do Uso de Plantas Medicinais na Vila Cachoeira, Ilhéus, Bahia, Brasil. Acta Farmacêutica Bonaerense, Buenos Aires, 21: 205-211.

MUELLER-DOMBOIS, D.; ELLENBERG, H. 1974. Aims and methods of vegetation ecology. New York: John Willey and Sons, 547p.

NOGUEIRA, N.O.; OLIVEIRA, O.M.; MARTINS, C.A.S.; BERNARDES, C.O. 2012. Utilização de leguminosas para recuperação de áreas degradadas. Enciclopédia Biosfera, Goiânia, 8: 21-31.

OHASHI, S.T.; LEÃO, N.V.M. 2005. Morototó: Schefflera morototoni (Aubl.) Maguire, Steyerm. \& Frodin. Belém: Informativo Técnico Rede de Sementes da Amazônia, n.12. 2p.

OKINO, E.Y.A.; SOUZA, M. R.; SANTANA, M.A.E.; SOUSA, M.E.; TEIXEIRA, D.E. 2004. Chapa Aglomerada de Cimento-madeira de Hevea brasiliensis Müll. Arg. Revista Árvore, Viçosa, 28: 451-457.

PARDO-DE-SANTAYANA, M.; MACÍA, M.J. 2015. The benefits of traditional knowledge. Nature, 518: 487-488.

PAULA, R.C.; PAULA, N.F. 2003. Sistemas Agroflorestais. In: VALERI, S.V.; POLITANO, W.; SENO, K.C.A.; BARRETO, A.L.N.M. (Org.). Manejo e Recuperação Florestal. São Paulo, Jaboticabal: Funep, 180p. 
REFLORA - HERBÁRIO VIRTUAL. Disponível em: <http://reflora.jbri.gov.br/reflora/ herbarioVirtual />. Acesso em 06/11/2016.

RIVERO, S.; ALMEIDA, O.; ÁVILA, S.; OLIVEIRA, W. 2009. Pecuária e desmatamento: uma análise das principais causas diretas do desmatamento na Amazônia. Nova Economia, Belo Horizonte, 19: 41-66.

RODRIGUES, F.Q. 2005. Composição florística, estrutura e manejo de sistemas agroflorestais no vale do rio Acre, Amazônia, Brasil. Dissertação de Mestrado, Universidade Federal do Acre, Rio Branco, Acre. 81p.

SALOMÃO, G.B.; PADOVAN, M.P.; PEREIRA, Z.V.; FERNADES, S.S.L.; MOITINHO, M.R. 2011. Uso múltiplo de espécies arbóreas em um sistema agroflorestal sob bases agroecológicas em Ponta Porã, Mato Grosso do Sul. In: Congresso Brasileiro de Sistemas Agroflorestais, 8. Belém. Anais... Belém, Embrapa Western Agriculture.

SANCHEZ, E.A. 1995. Science in agroforestry. Agroforestry Systems, 30: 5-55.

SANTELLI, P.; CALBO, M.E.R.; CALBO, A.G. 2006. Fisiologia pós-colheita de frutos da palmeira Syagrus oleracea (Mart.) Becc. (Arecaceae). Acta Botânica Brasilica, 20: 523-528.

SANTOS, S.R.M.; MIRANDA, I.S.; TOURINHO, M.M. 2004. Análise florística e estrutural de sistemas agroflorestais das várzeas do rio Juba, Cametá, Pará. Acta Amazônica, Manaus, 34: 251-263.

SCHARDONG, R.M.F.; CERVI, A.C. 2000. Estudos etnobotânicos das plantas de uso medicinal e místico na comunidade de São Benedito, Bairro São Francisco, Campo Grande, MS, Brasil. Acta Biológica Paranaense, Curitiba, 29: 187-217.

SHANLEY, P.; MEDINA, G. 2005. Frutíferas e Plantas Úteis na Vida Amazônica. 1ra ed. Belém: Cifor, Imazon, 300p.

SILVA, J.N.M.; LOPES, J.C.A.; OLIVEIRA, L.C.; SILVA, S.M.A.; CARVALHO, J.O.P.; COSTA, D.H.M.; MELO, M.S.; TAVARES, M.J.M. 2005. Diretrizes para a instalação e medição de parcelas permanentes em florestas naturais da Amazônia Brasileira. 1ra ed. Belém: Embrapa Amazônia Oriental, 68p.

SILVA, S.C. 2013. Sistemas agroflorestais na Amazônia: Fitossociologia, socioeconomia, análise de risco, comercialização de tendência de preços dos produtos. Tese de Doutorado, Universidade Federal de Lavras, Lavras, Minas Gerais. 213p.

SIPAM - GOVERNO DO ESTADO DE RONDÔNIA. 2006. Pedologia Rolim de Moura - RO. Porto Velho: Sipam, 1 Mapa, Escala 1:250.000.

SOUZA, C.R.; AZEVEDO, C.P.; ROSSI, L.M.B.; LIMA, R.M.B. 2008. Castanha-do-brasil (Bertholletia excelsa Humb. \& Bompl.). Manaus: Embrapa. 4p.

SOUZA, V.C.; LORENZI, H. 2008. Botânica sistemática; guia ilustrado para identificação das famílias de Angiospermas da flora brasileira, baseado em APG II. Nova Odessa: Instituto Plantarum, 640p. 
SOUZA, I.A. 2007. Avaliação de clones de seringueira (Hevea spp.) em Piracicaba-SP. Dissertação de mestrado, Escola Superior de Agricultura Luiz de Queiroz, Piracicaba, São Paulo, 71p.

TONINI, H.; ARCO-VERDE, M.F.; SÁ, S.P.P. 2005. Dendrometria de espécies nativas em plantios homogêneos no estado de Roraima - andiroba (Carapa guianensis Aubl), castanha-do-Brasil (Bertholletia excelsa Bonpl.), ipê-roxo (Tabebuia avellanedae Lorentz ex Griseb) e jatobá (Hymenaea courbaril L.). Acta Amazonica, 35: 353-362.

TURNER, M.G.; GARDNER, R.H.; O'NEILL, R.V. 2001. Landscape Ecology: in theory and practice. New York: Springer, 406p.

UHL, C.; MURPHY, P.G. 1981. Composition, Structure and Regeneration of a tierra firme Forest in the Amazonian Basin of Venezuela. Tropical Ecology, 22: 219-237.

VIEIRA, D.L.M.; HOLL, K.D.; PENEIREIRO, F.M. 2009. Agro-Successional Restoration as a Strategy to Facilitate Tropical Forest Recovery. Restoration Ecology, 17: 451-459.

VIEIRA, T.A. ROSA, L.S.; VASCONCELOS, P.C.S.; SANTOS, M.M.; MODESTO, R.S. 2007. Sistemas agroflorestais em áreas de agricultores familiares em Igarapé-Açu, Pará: caracterização florística, implantação e manejo. Acta Amazonica, Manaus, 37: 549-55.

VILHENA, M.R. 2004. Ciência, tecnologia e desenvolvimento na economia da castanha-do-brasil- A transformação industrial da castanha-do-brasil na COMARU-Região Sul do Amapá. Dissertação de mestrado, Universidade Estadual de Campinas, Campinas-SP, 149p.

WILSON, M.H.; LOVELL, S.T. 2016. Agroforestry-The next step in sustainable and resilient agriculture. Sustainability, $8: 15 p$. 